[Vicino Oriente XVI (2012), pp. 303-312]

\title{
UN’ANFORETTA DIPINTA DALLA TOMBA T.177 DI MOZIA
}

\author{
Federica Spagnoli - Sapienza Università di Roma
}

La tomba T.177 della Necropoli di Mozia ${ }^{1}$, una sepoltura ad incinerazione costituta da un'anfora utilizzata come urna e tre vasi di corredo ${ }^{2}$, ha restituito un'anforetta ${ }^{3}$, alta 14,8 $\mathrm{cm}$, con un diametro massimo di $15 \mathrm{~cm}$ e dell'imboccatura di $8,5 \mathrm{~cm}$; il vaso è contraddistinto da un profilo biconico con una coppia di anse a nastro desinenti sull'orlo a tesa orizzontale, collo troncoconico, spalla distinta arrotondata, corpo emisferico lievemente compresso; il piede è ad anello con rigonfiamento centrale e misura $5,8 \mathrm{~cm}$ di diametro (fig. 1).

Per la forma il vaso può essere ricondotto alle tradizioni ceramiche italiche ${ }^{4}$, in particolare al tipo di anforetta con decorazione a spirale di tradizione etrusco-laziale 5 . Questa produzione caratterizza i contesti etrusco-laziali e falisci di Età Orientalizzante, e costituisce una delle prime manifestazioni artigianali a carattere seriale ${ }^{6}$. Essa annovera forme biconiche con carenature e spigoli molto evidenti, caratterizzate da una particolare decorazione incisa in cui l'elemento più comune è, appunto, la doppia spirale sul ventre, e si colloca nella tradizione della ceramica d'impasto dell'Età del Ferro derivata da prototipi

1 Desidero ringraziare il Professor Lorenzo Nigro, direttore della Missione archeologica a Mozia dell’Università di Roma «La Sapienza» per avermi affidato lo studio e la pubblicazione dei materiali in oggetto.

2 Il corredo della tomba T.177 era costituito, oltre che dall'anforetta MN.11.T177/2, da un'anfora cinerario e da due olle monoansate. Si riporta di seguito la descrizione di questi vasi di corredo. Anfora cinerario MN.11.T177/1: conservata nella parte inferiore, ha corpo globulare, piede distinto ad anello, fondo con sezione ad onda; si conservano, inoltre, alcuni frammenti della spalla carenata e un'ansa a doppio bastoncello. L'anfora presenta una decorazione pittorica a linee parallele che inquadravano, probabilmente, uno schema metopale o un motivo a tremolo, secondo un modulo decorativo ampiamente attestato nei vasi di corredo della Necropoli Arcaica e della Collezione Whitaker. La decorazione è realizzata in vernice nera su una sottile velatura rosa chiaro, e si sviluppa nella parte mediana del vaso e, come mostrano alcuni frammenti non ricomponibili, anche sulla spalla. L'anfora è datata all'ultimo quarto dell’VIII secolo a.C. Olla monoansata con corpo globulare MN.11.T177/3: ricomposta in più frammenti, piccola parte dell’orlo mancante, con ampi confronti nei corredi delle tombe arcaiche (Tusa 1972, 34-36, 38-39, 79-81, tav. XXVII, T.3; 40, tav. XXXI:2, T.5). Olla monoansata con corpo globulare schiacciato MN.11.T177/4: conservata per metà. Simile a un esemplare dal corredo della tomba T.31 (Tusa 1972, 72, tav. LIV:2).

3 MN.11.T177/2.

4 Nel saggio di A.M. Bisi (1970, 17, 26, fig. 2) questo tipo di anforetta compare del repertorio della ceramica fenicio-punica (Forma 13) come derivata dal repertorio cipriota (Gjerstad 1948, fig. VI:1, 3, 5; fig. IX:5-6; fig. XI: 13; fig. XIV:6-7).

5 Bosio - Pugnetti 1986, 64, nn. 1, 2, tomba 90, secondo-terzo quarto VII sec. a.C. Le anforette in impasto di produzione ceretana (fine VIII-VII secolo a.C.) raggiungono un'ampia diffusione anche nel Lazio; proprio a causa della frequenza con cui la forma ricorre nelle necropoli laziali e di Roma agli inizi dell’Orientalizzante, è stata originariamente coniata per questa classe la denominazione di “anforette laziali” (Gambari 1980, 6869). Dal punto di vista della morfologia, l'esemplare moziese può essere confrontato con alcune anforette a spirali provenienti da Satricum: Waarsenburg 1995, 223-224, tav. 35, nn. 2.2-2.4, in particolare n. 2.2 per la forma estroflessa dell'orlo.

6 Benedettini 2007, 42-44. 
metallici ${ }^{7}$. L'area di produzione è quasi certamente da localizzarsi, almeno per il periodo di attestazione più antico (fine dell’VIII secolo a.C.), a Veio ${ }^{8}$ mentre, dagli inizi del VII secolo a.C., le principali officine di produzione e irradiazione possono essere individuate in Etruria Meridionale, in particolare a Cerveteri ${ }^{9}$.

Questa forma è già nota a Mozia dagli scavi della Necropoli Arcaica: Vincenzo Tusa rinvenne, tra i vasi di corredo della tomba T.82 ${ }^{10}$, un'anforetta tipologicamente riconducibile per profilo, scansione delle superfici e sezione delle anse, alla classe delle anfore con decorazione a spirali ${ }^{11}$ ma da attribuirsi, per il tipo di decorazione, a una produzione precedente (fig. 2). L'ornato della superficie, infatti, è costituito da una serie di fasci spezzati di sei linee disposte a zig-zag incisi e disposti nella parte centrale del corpo che sarà poi occupata dal motivo dominante della doppia spirale. L'anforetta della tomba T.82 trova numerosi confronti nei corredi nelle necropoli dell'Etruria Meridionale e del Latium Vetus dell'ultimo quarto - fine dell'VIII secolo a.C.12, ed è, con ogni probabilità, un vaso d'importazione ${ }^{13}$. La presenza dell'anforetta etrusco-laziale nel corredo della Tomba T.82 di Mozia è un fatto molto significativo per la complessiva rarità del tipo nei centri fenici e punici d'Occidente. I rinvenimenti sono circoscritti a pochissimi siti tra cui Mozia e Bithia in Sardegna ${ }^{14}$, probabilmente per il fatto che la produzione di queste anforette, nel periodo delle attestazioni nei due centri fenici, ha un raggio di diffusione ampio ma essenzialmente regionale, circoscritto alle aree di cultura etrusca e a quelle immediatamente limitrofe ${ }^{15}$. Le attestazioni in area fenicia potrebbero essere collegate a episodi singoli di contatto con queste produzioni, come suggerito da R. Zucca per il frammento bithiense, «un oggetto personale legato all'immigrazione di un etrusco nella città sarda» ${ }^{16}$.

7 Bosio - Pugnetti 1986, 89.

8 T. Dohrn $(1965,143-152)$ localizza tra Veio e Tarquinia i principali centri di produzione di questi esemplari per giustificare la loro ampia diffusione con la localizzazione delle officine in un grande scalo commerciale. Anfore a spirali sono anche attestate nei corredi pitecusani nell'ultimo quarto dell'VIII secolo a.C. (Rasmussen 1979, 70, tav. 2).

9 Nell'ultimo quarto dell'VIII secolo a.C. le anforette a spirali conoscono una vastissima diffusione tra il Lazio e l'Etruria in contesti sud-etruschi, falisci e laziali, grazie agli stetti contatti tra queste aree culturali (Colonna 1970, 641; (a cura di) 1976, 332, n. 19, tav. 88). La forma viene imitata in bucchero a partire dal secondo quarto del VII secolo a.C. (Beijer 1978, 11, tav. 4: 2-3; Bardelli 1980, 72-74, fig. V.1: a-c).

10 Tusa 1978, 28, tav. XVIII:3.

11 Bijer 1978, 11, tav. 4:2-3.

12 In particolare: tomba 520 della necropoli di Osteria dell’Osa, tomba principesca 872 da Veio-Casal del Fosso, tomba C della necropoli del Torrino, tomba 3 di Tor de’ Cenci, tomba XII della Riserva del Turiglio, tomba 1 rinvenuta sulle mura palatine di Roma (Benedettini 2007, 40, fig. 8, tav. 2, con bibliografia precedente).

13 Due frammenti di anforetta a spirali sono stati rinvenuti tra i materiali sconvolti all'interno del vano occidentale della Torre 4 delle mura di Mozia, e sono probabilmente da mettere in relazione con le tombe obliterate dalle strutture murarie del circuito difensivo (Ciasca 1979, 222, tav. LXXVIII:3; Spagnoli 20072008, 326-328, figg. 2-6).

14 Zucca 1985, 43-49.

15 Benedettini 2007, 44.

16 Zucca 1985, 48, note 50-51. 
Sia per la forma che per l'impasto e il trattamento superficiale, invece, l'anforetta della tomba T.177 può essere considerata un prodotto moziese ${ }^{17}$. La forma del vaso, infatti, presenta alcune varianti morfologiche rispetto agli esemplari di area etrusca, in particolare nella forma dell'orlo, a tesa orizzontale, e nelle proporzioni, conservando tuttavia un'aderenza formale al modello d'ispirazione. L'esemplare è di impasto depurato marroncino-rossastro e presenta sulla superficie esterna un rivestimento beige sottile ben conservato. La decorazione è dipinta in nero e si dispone nella parte superiore del vaso su orlo, collo e anse. Sulla tesa dell'orlo i tratti dipinti, radiali rispetto alla circonferenza, sono spezzati al centro da un filetto anulare; sulle anse sono invece una serie di tratti orizzontali posti a distanza regolare gli uni dagli altri. Sul collo e sul corpo del vaso il motivo decorativo principale è costituito dalla linea ondulata o "tremolo" verticale, ripetuto per sei volte in moduli da quattro e che, partendo dall'orlo, percorre la lunghezza del collo fino ad oltrepassare la carena che distingue il passaggio dal collo al corpo, terminando appena sotto la linea dell'imposta delle anse. I tremoli sono delimitati in basso da due linee concentriche sottili.

I motivi decorativi presenti sul vaso in esame sono piuttosto comuni nella tradizione della ceramica fenicia e in particolare in quella moziese ${ }^{18}$. Il tipo di decorazione lineare, nelle varianti a tratti radiali, linee parallele o cerchi concentrici, è presente nel repertorio della ceramica dipinta per tutto il periodo arcaico fino alla prima metà del VI secolo a.C.19. Le composizioni decorative che sfruttano il motivo base del tremolo, presente sui vasi moziesi, sia in orizzontale singolo che in gruppi composti da più linee di metope verticali, sono invece attestate nelle ceramiche più antiche provenienti dalla necropoli e in alcuni vasi della Collezione Whitaker ${ }^{20}$. In questi la linea ondulata orizzontale è generalmente impiegata singolarmente sul corpo del vaso per riempire una parte libera o inquadrata da

17 Dalla tomba T.11 della Necropoli Arcaica proviene un'anforetta riconducibile per forma quelle etrusco-laziali in impasto (Tusa 1972, 46, tav. XXXIV:2) ma che, nella fattura e nella decorazione a fasce in Red-Slip nella parte di massimo diametro del corpo, è certamente da attribuirsi a una produzione locale. Tra i vasi di corredo di una tomba rinvenuta nel 1921 è presente un'anforetta dipinta realizzata a ruota lenta che presenta dei tratti in comune con l'esemplare della tomba T.177. L'anforetta (fig. 3) ha una forma globulare schiacciata con profilo continuo, orlo estroflesso e anse a sezione circolare tra orlo e spalla. La decorazione dipinta in bruno si dispone sull'orlo con brevi tratti radiali, e sulle anse, in maniera analoga all'altra, mentre sul corpo consiste in un elemento a zig-zag alla base del collo, e in un tremolo continuo campito poco al di sopra della massima espansione del corpo, in corrispondenza delle quale è una linea orizzontale (S. Tusa 2008, 77, n. 19, tav. IV).

18 I primi studi sul repertorio ceramico dipinto moziese si devono a A.M. Bisi che ha trattato l'argomento in diversi saggi tra cui si ricordano, oltre il già citato del 1970, l'articolo pubblicato nel 1968 su Africa ( Bisi 1968a) e quello uscito nello stesso anno su Studi Magrebini (Bisi 1968b), cui si aggiungono lo studio specifico sui repertori dipinti della Sicilia fenicio-punica su Sicilia Archeologica (Bisi 1969) e il saggio generale presentato al congresso sulla colonizzazione di Barcellona del 1971 e pubblicato negli Atti nel 1974

19 Bisi 1969, 14. La decorazione lineare sul dorso dell'ansa è attestata, pur con minore frequenza, anche nei vasi di V secolo a.C.

20 Il motivo lineare e quello a tremolo compaiono spesso associati anche in schemi complessi, come ad esempio su una particolare oinochoe trilobata, nella quale fasce orizzontali di diversi spessori dipinte sul collo e sulla pancia si alternano a serie oblique di tremoli sulla spalla e orizzontali sul collo. La decorazione di questo vaso di corredo della tomba T.156 della necropoli moziese (Tusa 1978, 60, tav. XLVII:1) è ispirata probabilmente alle produzioni greco-orientali, poiché mostra analogie con una brocca di fabbrica euboica rinvenuta tra i vasi di corredo di una necropoli di Pithecusa (Buchner 1964, 263-274; Bisi 1969, 15, fig. 7). 
bande di pittura ${ }^{21}$, ed è spesso associata ad una decorazione lineare regolare del collo e delle anse; altrettanto frequentemente è utilizzata come elemento divisorio di motivi geometrici in composizioni metopali ${ }^{22}$. Nel repertorio vascolare della necropoli questi motivi si ritrovano principalmente su forme chiuse, anche se non mancano esempi di decorazione a tremolo su coppe e skyphoi ${ }^{23}$. La semplice fascia sinuosa orizzontale, ampiamente diffusa anche nelle serie geometriche greche, è un motivo presente nella ceramica euboica ${ }^{24}$, e compare frequentemente in associazione ad una coppia di linee nel repertorio corinzio tardo-geometrico e in quello del Protocorinzio Antico ${ }^{25}$. Tuttavia, sebbene questo sia stato spesso misconosciuto, questi tipi di decorazione, a tratti paralleli e radiali e a linea ondulata continua, hanno profonde radici nelle produzioni dipinte del Levante sin dal II millennio a.C. e vennero rinverdite dal contatto con alcune produzioni dipinte cipriote ${ }^{26}$. In tal modo questi precursori si debbono tenere presenti come elementi appartenenti al background ceramico fenicio che favorirono la ricezione di tradizioni più direttamente mediterranee il cui consolidamento, nei repertori decorativi di Occidente, è stato reso effettivo dalla mediazione corinzia ${ }^{27}$.

Per quanto riguarda la disposizione della decorazione nell'esemplare in esame, si può osservare come questa tenga solo marginalmente conto della tettonica del vaso, in particolare per i tremoli verticali che, dal limite superiore del collo, si sviluppano in lunghezza fin oltre la linea della carenatura alla base di questo, terminando con una cornice composta da due linee parallele che non trova corrispettivo nella parte superiore. Anche

21 Si vedano l'anfora della tomba T.172 (MM78/163) e l'anfora della tomba T.167, MM78/133/78-25, obliterate dalla Torre 4 delle mura: Ciasca 1979, 210-211, 213-215; Spagnoli 2007-2008, figg. 3-4.

22 Gli schemi decorativi complessi a motivi metopali non sembrano andare oltre la fine del VII secolo a.C. e sono attestati soltanto sui vasi della necropoli, mentre quelli dagli strati più antichi del tofet presentano principalmente una decorazione lineare: Bisi 1969, 14; Famà - Toti 2005, 618, figg. 5-6. Si riporta di seguito la descrizione di tre vasi appartenenti alla Collezione Whitaker che sono esemplificativi della varietà del repertorio decorativo moziese: brocca neck-ridge N.I. 2720, dal tofet (Ciasca 1978, tav. LXXIII:4, 6; 1979, 211, nota 14, fig. 16:5): sul corpo un fregio formato da un tremolo orizzontale delimitato in alto e in basso da due linee concentriche; decorazione lineare sul collo. Anfora N.I. 2753, dalla necropoli (Cintas 1970, tav. XXXIV, fig. 103; Bisi 1969, 13, fig. 5:10): decorazione a registri sovrapposti delimitati da linee sottili. Nella parte centrale è una decorazione metopale composta da triglifi e losanghe su lungo stelo, motivo ripetuto anche nel registro superiore. Sulla spalla, ancora gruppi di losanghe e tremoli orizzontali. Anfora N.I. 2755, dalla necropoli (Bisi 1969, 13, fig. 5:11): collo e orlo mancanti, sulla spalla cinque gruppi di quattro tremoli verticali posti a distanza regolare, alternati a disegno romboidale/quadrato quadrettato. Sotto, nello spazio compreso tra le anse, decorazione metopale composta da triglifi di tremoli verticali e metope vuote. Il fregio è delimitato in alto e in basso da due linee parallele, sulle anse (orizzontali) è una decorazione a tratti verticali.

23 Famà - Toti 2005, 618, fig. 6 (inizi VII secolo a.C.).

24 Canciani 1975, 82.

25 Canciani 1974, 25, tav. 18:2. Il tremolo verticale moltiplicato e disposto all'interno di un registro riprende uno schema comune anche ad alcune serie beotiche costituite per di più da anfore (Ruckert 1976, 56, fig. 23:a), per le quali sono state riconosciute le connessioni con le produzioni euboico-cicladiche, in cui questo motivo è molto diffuso (Canciani 1974, 209).

26 Aubet - Núñez 2008, 86-93.

27 Il motivo a linea ondulata, infatti, è presente nelle produzioni corinzie a partire dal Protocorinzio Antico (Coldstream 1968, 106, tav. 21:k), ma formulazioni simili compaiono anche nelle serie geometriche euboicocicladiche (Coldstream 1968, 175, tav. 41:f) e più tardi nella produzione protoattica (Kubler 1959, 115, fig. 5; Neri 2010, 186-187). 
sulla tesa dell'orlo i gruppi di trattini radiali superano la larghezza della tesa andando a cadere nella parte superiore delle anse. La stessa libertà nella posizione dei motivi decorativi si riscontra su altri vasi dipinti moziesi ${ }^{28}$. Per questo tema Antonia Ciasca aveva coniato la definizione di "fenicio-geometrico" 29 per sottolinearne la derivazione dalle produzioni greche inquadrando il fenomeno all'interno della ricezione dei modelli greci da parte delle colonie fenicie ${ }^{30}$. La ceramica dipinta moziese, infatti, serba con più fedeltà, rispetto alla stessa Cartagine, l'impronta della sintassi decorativa del geometrico greco, forse proprio per l'assiduità dei contatti e degli scambi con le popolazioni indigene e con i coloni greci delle città della Sicilia Occidentale. Questa influenza ha come effetto la distribuzione degli elementi decorativi sull'intera superficie del vaso, come per uno horror vacui che non trova riscontro nel repertorio pittorico degli altri centri fenici d'Occidente ${ }^{31}$.

Nell'anforetta della tomba T.177 l'irregolarità della disposizione della pittura diventa cifra stilistica, caratterizzata da un'estrema libertà di composizione solo parzialmente vincolata da uno schematismo di ascendenza greca. Questo è il frutto della rielaborazione in forme originali, da parte della cultura moziese, dei motivi geometrici e lineari che costituiscono i modelli diretti di riferimento, attraverso il recupero quelle peculiarità che affondano le radici nella più antica tradizione decorativa del Mediterraneo Orientale.

\section{BIBLIOGRAFIA}

AUBET, M.E. - NÚÑEZ, F.J.

2008 Cypriot imports from the Phoenician cemetery of Tyre al-Bass: C. DOUMET-SERHAL (ed.), Networking Patterns of the Bronze and Iron Age Levant. The Lebanon and its Mediterranean Connections. On the Occasion of the Symposium "Interconnections in Eastern Mediterranean: the Lebanon in the Bronze and Iron Ages”, 4-9 November 2008, Beirut (Archaeology and History in Lebanon), Beirut 2008, pp. 71-104.

BARDELLI, O.J.

1980 Il Bucchero: M. BongHI Jovino (a cura di), Gli etruschi e Cerveteri. Nuove acquisizioni delle Civiche Raccolte Archeologiche. La prospezione archeologica nell'attività della fondazione Lerici. Milano, Palazzo Reale, Settembre 1980-Gennaio 1981, Milano 1980, pp. $72-80$.

28 Ad esempio su un'anfora della Necropoli Arcaica proveniente dalla tomba T.163 (Tusa 1978, 62, tav. XLIX:3): il cinerario presenta una decorazione metopale a tremoli e file di puntini alternati che, partendo dalla carena che distingue la spalla dal corpo, ricade sotto il punto di attacco delle anse, dove è interrotta da due linee parallele, in maniera del tutto analoga al nostro esemplare. Il superamento dei limiti imposti dalla tettonica del vaso è tuttavia più evidente nell'anforetta della tomba T.177, dove il motivo geometrico si dispone con maggiore libertà.

29 Ciasca 1999, 71.

30 Sul geometrico siciliano, e in particolare a Mozia, si vedano i saggi di P. Orsi (1898, 305 segg.), B. Pace (1915, 441) e J.I.S. Whitaker (1921, 221-225).

31 Bisi 1974, 23. 
BENEDETTINI, M.G.

2007 Le produzioni ceramiche medio-italiche di Età Orientalizzante: G. BARTOLONI (a cura di), Il Museo delle Antichità Etrusche e Italiche. II. Dall'incontro con il mondo greco alla romanizzazione, Roma 2007, pp. 31-134.

BIJER, A.J.

1978 Proposta per una suddivisione delle anfore a spirali: Mededelingen van het Nederlands Institut te Rome 40 (1978), pp. 7-21.

BISI, A.M.

1968a La ceramica di tradizione fenicio-punica della Sicilia occidentale: Africa II (1968), pp. 1135.

1968b Aspetti e problemi della ceramica punica arcaica dipinta: Studi Magrebini 2 (1968), pp. 143.

1969 La ceramica a decorazione dipinta della Sicilia fenicio-punica: Sicilia Archeologica 6 (1969), pp. 11-20.

$1970 \quad$ La ceramica punica. Aspetti e problemi, Napoli 1970.

1974 Le componenti mediterranee e le costanti tipologiche della ceramica punica: E. RIPOLL Perelló (ed.), Simposio Internacional de Colonizaciones, Barcelona 1971, Barcelona 1974, pp. 15-23.

Bosio, B. - PugnetTi, A.

1986 Gli Etruschi di Cerveteri. La necropoli di Monte Abatone, tombe 32-45-76-77-79-81-8389-90-94-102, Modena 1986.

BUCHNER, G.

1964 Dibattito: AA.VV., Metropoli e colonie di Magna Grecia. Atti del III Congresso di Studi CANCIANI, F. sulla Magna Grecia, Taranto 13-17 ottobre 1963, Napoli 1964, pp. 263-274.

1974 CVA, Tarquinia, Museo Nazionale, 3. Italia 55, Roma 1974.

1975 Un Biconico dipinto da Vulci: Dialoghi d’Archeologia 8 (1975), pp. 79-85.

CIASCA. A.

1978 Mozia 1977. Scavi alle mura (campagna 1977): Rivista di Studi Fenici VI (1978), pp. 227-245.

1979 Scavi alle mura di Mozia (campagna 1978): Rivista di Studi Fenici VII (1979), pp. 207227.

1999 Sicilia e Malta. Note su repertori ceramici a confronto: A. GonzÁles PRATs (ed.), La cerámica fenicia en Occidente: centros de producción y áreas de comercio. Actas del I Seminario Internacional sobre Temas Fenicios, Guardamar del Segura, 21-24 de noviembre de 1997, Alicante 1999, pp. 69-87.

CINTAS, P.

1970 Manuel d'archéologie punique I. Collection des Manuels d'Archéologie et d'Histoire de l'Art, Paris 1970.

COLDSTREAM, J.N.

1968 Greek Geometric Pottery, London 1968.

COLONNA, G.

1970 Una nuova iscrizione etrusca del VII secolo e appunti sull'epigrafia ceretana dell'epoca: ColonNa, G. (a cura di)

1976 Civiltà del Lazio Primitivo. Palazzo delle Esposizioni, Roma 1976, Roma 1976

DOHRN, T.

1965 Die etruskische Bandhenkelamphoren des 7. Jh. v.C.: R. BIANCHI BANDiNELLI (a cura di), Studi in onore di Luisa Banti, Roma 1965, pp. 143-152. 
FAMÀ, M.L. - TOTI, M.P.

2005 Materiali inediti dalla collezione 'G. Whitaker’ di Mozia: A. SPANÒ GIAMmELlaro (a cura di), Atti del V Congresso Internazionale di Studi Fenici e Punici (Marsala-Palermo 2-8 ottobre 2000), Palermo 2005, pp. 615-630.

GAMBARI, F.M.

1980 Osservazioni sulle anforette: M. Bonghi Jovino (a cura di), Gli etruschi e Cerveteri. Nuove acquisizioni delle Civiche Raccolte Archeologiche. La prospezione archeologica nell'attività della fondazione Lerici. Milano, Palazzo reale, Settembre 1980-Gennaio GJERSTAD, E. 1981, Milano 1980, pp. 68-69.

1948 The Swedish Cyprus Expedition IV, 2, Stockholm 1948

KUBLER, K.

1959 Kerameikos VI:1-2. Die Nekropole des späten 8. bis frühen 6. Jahrhunderts, Berlin 1959.

NERI, S.

2010 Il tornio e il pennello. Ceramica depurata di tradizione geometrica di epoca orientalizzante in Etruria Meridionale (Veio, Tarquinia, Vulci) (Officina Etruscologia 2), Roma 2010

ORSI, P.

1898 Le necropoli di Licodia Eubea ed i vasi geometrici del quarto periodo siculo: Römischer Mitteilungen 13 (1898), pp. 305-366.

PACE, B.

1915 Prime note sugli scavi di Mozia: Notizie degli Scavi di Antichità 12 (1915), pp. 431-446.

RASMUSSEN, T.B.

1979 Bucchero Pottery from Southern Etruria, Cambridge 1979.

RÜCKERT, A

$1976 \quad$ Frühe keramik Bootiens: Antike Kunst (Beiheft 10), Basel 1976.

SPAGNOLI, F.

2007-2008 Sepolture intramurali a Mozia: G. BARToloni - M.G. BenedetTini (a cura di), Atti del convegno internazionale "Sepolti tra $i$ vivi: evidenza ed interpretazione di contesti funerari in abitato”, Università di Roma «La Sapienza», 26-29 aprile 2006 (Scienze delle Antichità 14), Roma 2007-2008, pp. 323-346.

TUSA, S.

2008 Gli elementi di interesse paletnologico nella Collezione Whitaker e la preistoria moziese: R. De Simone - M.P. Toti (a cura di), La Collezione Whitaker. Volume I, Palermo 2008, pp. 67-85.

TUSA, V.

1972 La Necropoli Arcaica e adiacenze. Lo scavo del 1970: F. BEviLACQUA - A. CiAsCA - G. Matthiae SCANDOne - S. Moscati - V. Tusa - A. Tusa Cutroni, Mozia - VII. Rapporto preliminare della Missione congiunta con la Soprintendenza alle Antichità della Sicilia Occidentale (Studi Semitici 40), Roma 1972, pp. 7-81.

1978 La Necropoli Arcaica e adiacenze. Relazione preliminare degli scavi eseguiti a Mozia negli anni 1972, 1973, 1974: A. CiASCA - G. COACCI POLSELli - N. CUOMO DI CAPRIO M.G. Guzzo Amadasi - G. Matthiae Scandone - V. Tusa - A. Tusa Cutroni - M.L. Uberti, Mozia - IX. Rapporto preliminare della Missione congiunta con la Soprintendenza alle Antichità della Sicilia Occidentale (Studi Semitici 50), Roma 1978, pp. 7-98. 
WAARSENBURG, D.J.

1995 The Northwest Necropolis of Satricum. An Iron Age Cemetery in Latium Vetus (Scrinium, Monographs on History, Archaeology and Art History, VIII. Satricum, Reports and Studies of the Satricum Project III), Amsterdam 1995.

WITHAKER, J.I.S.

1921 Motya: a Phoenician colony in Sicily, London 1921

ZUCCA, R.

1985 Un frammento di anforetta a doppia spirale d'impasto da Bithia: Studi Etruschi 53 (1985), pp. 43-49. 


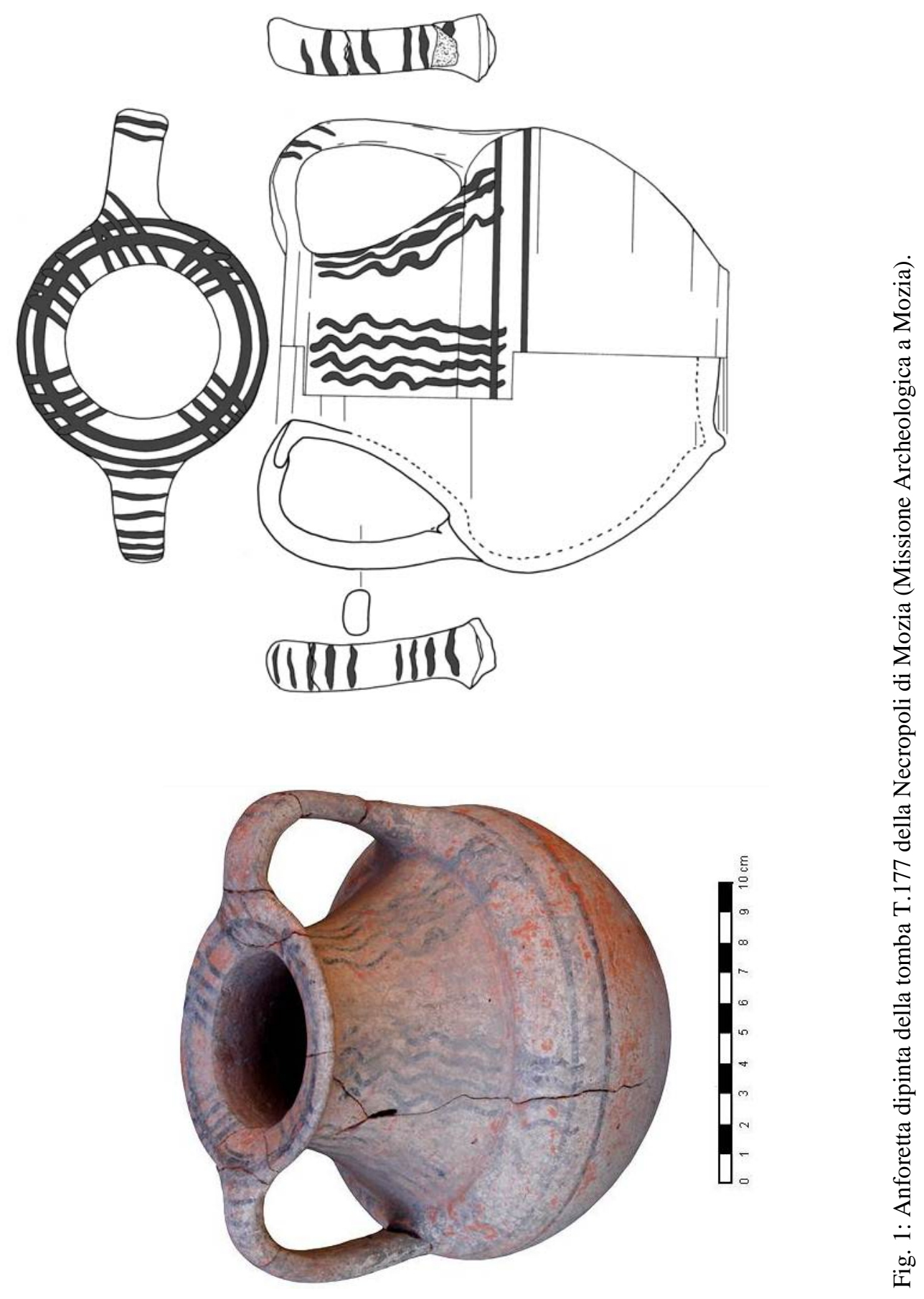



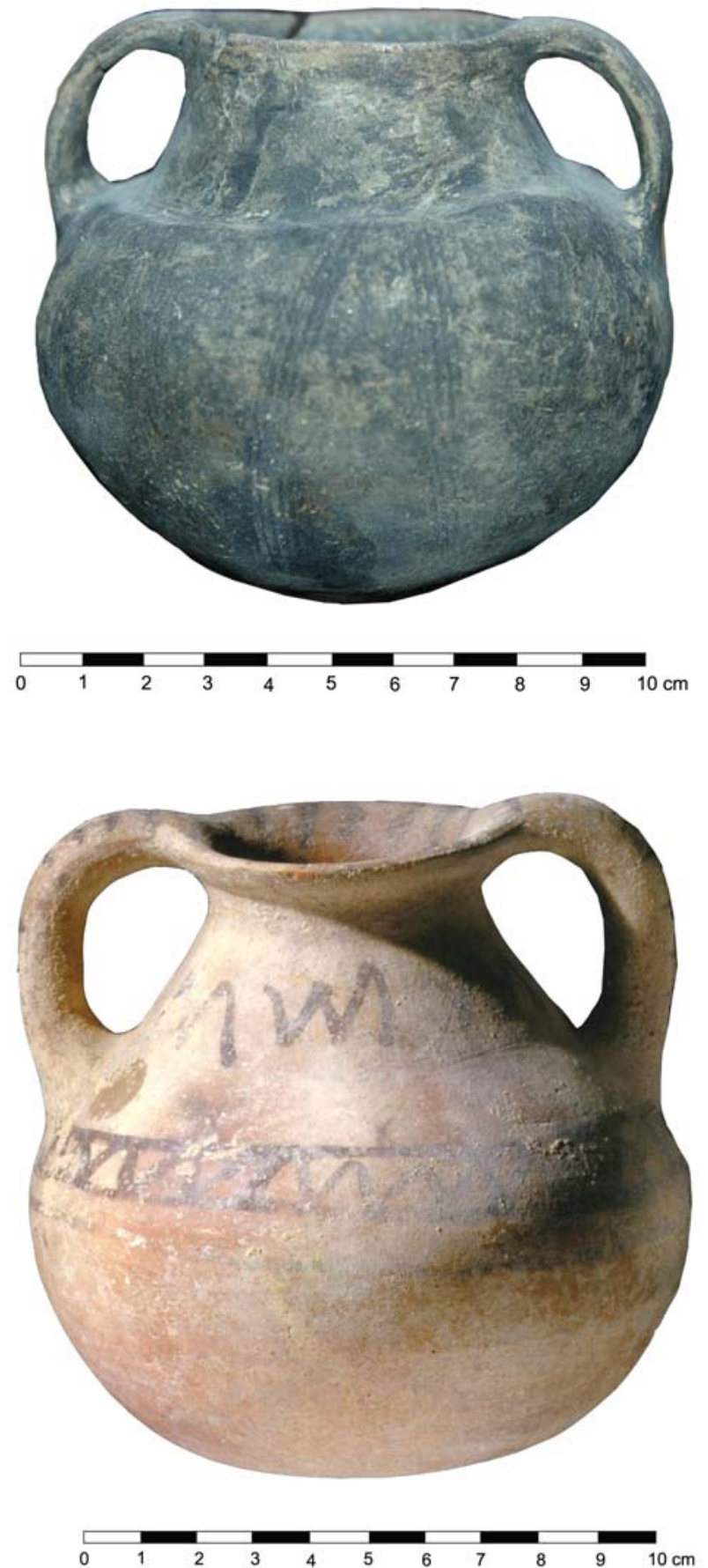

Fig. 2: Anforetta etrusco-laziale della tomba T.82 della Necropoli Arcaica (foto Museo Whitaker).

Fig. 3: Anforetta dipinta, Collezione Whitaker (da S. Tusa 2008, tav. IV:19). 